\title{
Investigation of the Endoplasmic Reticulum Localization of UDP- Glucuronosyltransferase 2B7 with Systematic Deletion Mutants ${ }^{\text {S }}$
}

\author{
Yuu Miyauchi, Sora Kimura, Akane Kimura, Ken Kurohara, Yuko Hirota, Keiko Fujimoto, \\ Peter I. Mackenzie, Yoshitaka Tanaka, and (D) Yuji Ishii \\ Division of Pharmaceutical Cell Biology, Graduate School of Pharmaceutical Sciences (Y.M., A.K., K.K., Y.H., K.F., Y.T.) and \\ Laboratory of Molecular Life Sciences, Graduate School of Pharmaceutical Sciences (Y.M., S.K., Y.I.), Kyushu University, \\ Fukuoka, Japan; and Department of Clinical Pharmacology, Flinders Medical Centre and Flinders University, Adelaide, South \\ Australia, Australia (P.I.M.)
}

Received August 10, 2018; accepted March 5, 2019

\begin{abstract}
UDP-Glucuronosyltransferase (UGT) plays an important role in the metabolism of endogenous and exogenous compounds. UGT is a type I membrane protein, and has a dilysine motif $(K K X X / K X K X X)$ in its C-terminal cytoplasmic domain. Although a dilysine motif is defined as an endoplasmic reticulum (ER) retrieval signal, it remains a matter of debate whether this motif functions in the ER localization of UGT. To address this issue, we generated systematic deletion mutants of UGT2B7, a major human isoform, and compared their subcellular localizations with that of an ER marker protein calnexin (CNX), using subcellular fractionation and immunofluorescent microscopy. We found that although the dilysine motif functioned as the ER retention signal in a chimera that replaced the cytoplasmic
\end{abstract}

domain of CD4 with that of UGT2B7, UGT2B7 truncated mutants lacking this motif extensively colocalized with CNX, indicating dilysine motif-independent ER retention of UGT2B7. Moreover, deletion of the C-terminal transmembrane and cytoplasmic domains did not affect ER localization of UGT2B7, suggesting that the signal necessary for ER retention of UGT2B7 is present in its luminal domain. Serial deletions of the luminal domain, however, did not affect the ER retention of the mutants. Further, a cytoplasmic and transmembrane domain-deleted mutant of UGT2B7 was localized to the ER without being secreted. These results suggest that UGT2B7 could localize to the ER without any retention signal, and lead to the conclusion that the static localization of UGT results from lack of a signal for export from the ER.
This study was supported in part by the Japan Research Foundation for Clinical Pharmacology; a Grant-in-Aid for Scientific Research (B) [Grant \#25293039] from the Japanese Society of Promotion of Science to Y.I.; Qdai-jump Research Program Wakaba Challenge [Grant \#30215] from Kyushu University to Y.M.; and a Grant-in-Aid for Scientific Research (C) [Grant \#19590147] from the Ministry of Science, Education, Sports and Technology to Y.I. The authors declare that the research was conducted in the absence of any commercial or financial relationships that could be construed as a potential conflict of interest.

Part of this work was presented as follows: 28th Annual Meeting of the Japanese Society of the Study of Xenobiotics, 2013 Oct 9-11, Tokyo, Japan. The Japanese Society of the Study of Xenobiotics, Tokyo, Japan. (Kimura S, Miyauchi Y, Mackenzie PI, Yamada H, Ishii Y, The C-terminal double lysine motif of UDP-glucuronosyltransferase 2B7 is not essential for its retention in endoplasmic reticulum); 134th Annual Meeting of the Japanese Society of Pharmaceutical Sciences, 2014 Mar 27-30, Kumamoto, Japan. The Japanese Society of Pharmaceutical Sciences, Tokyo, Japan (Kimura S, Miyauchi Y, Hirota Y, Tanaka Y, Mackenzie PI, Yamada H, Ishii Y, Role of the luminal domain of UDP-glucuronosyltransferase $2 \mathrm{~B} 7$ as an endoplasmic reticulumretention signal); Joint Meeting of the 19th North American International Society for the Study of Xenobiotics and 29th Annual Meeting of the Japanese Society for the Study of Xenobiotics, 2014 Oct 19-23, San Francisco, CA. The International Society for the Study of Xenobiotics, Washington DC; and The Japanese Society of the Study of Xenobiotics, Tokyo, Japan (Kimura S, Miyauchi Y, Mackenzie PI, Yamada H, Ishii Y, Mechanism for the retention of UDP-glucuronosyltransferase 2B7 in the endoplasmic reticulum: the role of the luminal domain); and Forum 2017: Pharmaceutical Health Care and Environmental Toxicology, 2017 Sep 1-2, Sendai, Japan. The Pharmaceutical Society of Japan, Tokyo, Japan (Miyauchi Y, Kimura S, Fujimoto K, Hirota Y, Mackenzie PI, Ishii Y, Tanaka Y, Determination of a novel targeting signal of UGT2B7 for its localization to the ER membrane).

Y.M. and S.K. contributed equally to this work.

https://doi.org/10.1124/mol.118.113902.

S This article has supplemental material available at molpharm. aspetjournals.org.

\section{Introduction}

In eukaryotic cells, the secretory pathway is a conserved biosynthetic transport route for secreted proteins and proteins localized in each organelle constituting the pathway. All of the proteins transported in this pathway are synthesized in the endoplasmic reticulum (ER) and delivered to their destinations by vesicular transport (Gomez-Navarro and Miller, 2016). In general, the following two strategies are believed to be necessary for ER proteins to reside in the ER: 1) retention (elimination from the vesicles involved in anterograde transport); and 2) retrieval [capture and return of the escaped ER residents by coat protein I complex (COPI)] vesicles (Teasdale and Jackson, 1996; Barlowe and Helenius, 2016). The mechanism of retention remains controversial, but the second mechanism is more established and is known as motif-based retrieval to the ER. The carboxyl-terminal tetrapeptides KDEL and HDEL are necessary for ER-soluble proteins to be retrieved by COPI in mammals and yeast, respectively (Munro and Pelham, 1987; Pelham, 1988). The C-terminal dilysine motif (DM) (KKXX/KXKXX) is also defined as a retrieval signal for type I membrane residents in the ER (Nilsson et al., 1989; Jackson et al., 1993; Gaynor et al., 1994).

UDP-Glucuronosyltransferase (UGT) is one of the major drug-metabolizing enzymes catalyzing glucuronidation, and 22 isoforms have been identified so far. Among them, 19 isoforms have been reported to be involved in glucuronidation in humans (Rowland et al., 2013). Most research on UGT is 
focused on its enzymatic activity, because the enzyme is involved in the metabolism of the second largest number of drugs, following cytochrome P450 (Evans and Relling, 1999). However, UGT is also a model for studies on ER localization. UGT is a typical type I membrane protein, with most of the protein, including the substrate-binding site, on the luminal side of the ER membrane. Only a single transmembrane (TM) helix exists in its C-terminal region, followed by about 20 residues facing the cytosol (cytosolic tail, CT). UGT has a DM at its C-terminus (Fig. 1) (Iyanagi et al., 1986; Harding et al., 1987; Shepherd et al., 1989; Radominska-Pandya et al., 1999), and its functions were well examined. The DM of UGT was transplanted onto the end of the CT of plasma membrane proteins (e.g., CD4, CD8, and ErbB2), which altered their localization from the plasma membrane to the ER (Jackson et al., 1990; Kinosaki et al., 1993). These studies were enough to believe that the C-terminal motif was a major driving force for UGT localization in the ER. However, there is also evidence that UGT is able to localize in the ER in a DM-independent manner. Deletion mutants of rat UGT2B1, human UGT1A6, and other isoforms lacking the CT and TM region are still retained in the ER (Jackson et al., 1993; Meech et al., 1996; Ouzzine et al., 1999). In our recent study, we also obtained similar results with UGT2B7. UGT2B7 is an important human isoform catalyzing the metabolism of a huge number of clinical drugs (e.g., morphine and zidovudine) (Rowland et al., 2013). In addition, UGT2B7 is also involved in the glucuronidation of endogenous compounds such as fatty acids, and steroids (Bowalgaha et al., 2007; Bock, 2012). Further, there are reports that UGT can localize to other organelles beside the ER (e.g., mitochondria, Golgi apparatus, and plasma membrane) (Chowdhury et al., 1985; Radominska-Pandya et al., 2005; Ménard et al., 2013; Ziegler et al., 2015). A growing number of studies demonstrates that the ER can make contact with other organelles, exchange membrane composition, and function as a central hub for organelle interaction (Schrader et al., 2015; Shim, 2017). Thus, elucidating the mechanisms by which UGT localizes to the ER is expected to lead to new insights into drug metabolism.

To address this issue, in the present study, we used two separate procedures (cellular fractionation and immunocytochemistry) to ascertain the role of the DM in UGT2B7 localization. We then generated systematic deletion mutants lacking not only the C-terminal TM and/or cytoplasmic domains, but also one or several sections of the ER luminal domain of UGT2B7 to look for another ER retention sequence.

\section{Materials and Methods}

Materials. Synthetic oligonucleotides were purchased from Fasmac (Kanagawa, Japan). Restriction enzymes and other DNA-modifying enzymes were from Takara Bio (Shiga, Japan). Glass products for immunofluorescence microscopy were from Matsunami Glass (Osaka, Japan). Pooled human liver microsomes (HLM; prepared from 50 donors) were from Corning Gentest (Woburn, MA). All other reagents were of the highest quality commercially available.

Subcloning of UGT2B7 and Its Mutants into a Bacuroviral Vector. Recombinant baculoviruses coding UGT2B7 wild-type (WT), $\Delta \mathrm{CT}(\Delta 511-529), \Delta \mathrm{TM}(\Delta 493-529)$, and human calnexin $(\mathrm{CNX})$ were

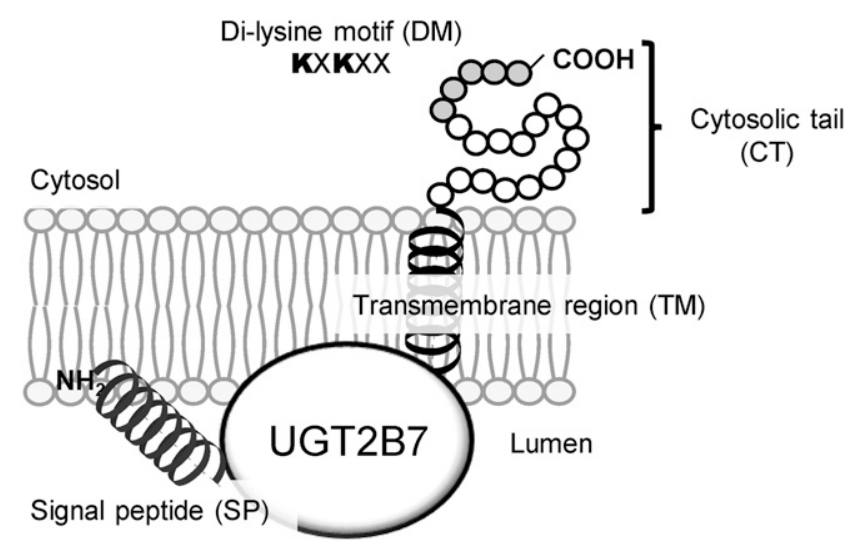

Fig. 1. Postulated topology of UGT2B7 in the ER membrane.

prepared using Bac-to-Bac Baculovirus Expression System (Life Technologies, Carlsbad, CA) as described previously (Miyauchi et al., 2015). $\Delta \mathrm{DM}(\Delta 525-529)$ was generated by polymerase chain reaction (PCR) using pFastBac1-WT as a template, KOD-Plus-Neo DNA polymerase (Toyobo Life Science, Osaka, Japan), and the following a pair of primers: sense, 5 -GGGGTACCGGGATGTCTGTTAAATGG-3'; anti-sense, 5'-GGGGTACCTTACTTTGCTTTTCTAGCAAA-3' (underlining indicates KpnI sites). Thermal cycle parameters were as follows: initial denaturation: $94^{\circ} \mathrm{C}, 2$ minutes; cycling step ( $\times 40$ rounds): $98^{\circ} \mathrm{C}, 10$ seconds; $52^{\circ} \mathrm{C}, 30$ seconds; $68^{\circ} \mathrm{C}, 1$ minute; hold, $4^{\circ} \mathrm{C}$. The PCR product was purified with FastGene Gel/PCR Extraction Kit (NIPPON Genetics, Tokyo, Japan) and subcloned into $\mathrm{pFastBac1}$ vector using $\mathrm{KpnI}$ sites. Hemagglutinin (HA) epitope tag (HA-tag) was introduced into the C-terminus of WT to yield WT-HA by QuickChange site-directed mutagenesis. Primers were designed by Agilent QuickChange Primer Design Program (https://www.genomics. agilent.com/primerDesignProgram.jsp), and non-tagged pFastBac1 construct was used as a template. Sequences of the primers were as follows: sense, 5' - GCTAGAAAAGCAAAGAAGGGAAAAAATGATTACCCATACGATGTTCCAGATTACGCTTAGGGTACCAAGCTTG-3'; anti-sense, 5' CAAGCTTGGTACCCTAAGCGTAATCTGGAACATCGTATGGGTAATCATTTTTTCCCTTCTTTGCTTTTCTAGC-3' (underlining indicates coding HA-tag). Thermal cycle parameters were "a two-step cycle" as follows: initial denaturation, $94^{\circ} \mathrm{C}, 2$ minutes; cycling step ( $\times 20$ rounds): $98^{\circ} \mathrm{C}, 10$ seconds; $68^{\circ} \mathrm{C}, 4$ minutes; hold, $4^{\circ} \mathrm{C}$. Template vectors were digested by DpnI treatment, and the reaction mixtures were used in transformation with Competent Quick DH5 $\alpha$ (Toyobo, Life Science Department, Osaka, Japan). Further deletion mutants with HA-tag ( $\Delta 1$-HA to $\Delta 5$-HA) were also prepared by site-directed mutagenesis using the pFastBac-WT-HA construct as a template. Primers are listed in Supplemental Table 1. Mutagenesis was carried out under the two-step cycling mentioned above. The nucleotide sequences of the constructs were confirmed by an Applied Biosystems 3130xl Genetic Analyzer (Thermo Fisher Scientific, Waltham, MA), using a BigDye Terminator version 3.1 Cycle Sequencing Kit (Life Technologies). Recombinant pFastBac1 were transfected into competent Escherichia coli DH10Bac strain (Life Technologies). After blue/white selection, a positive single clone was picked up and cultured to obtain recombinant bacmid, a part of baculoviral DNA, according to the user manual.

Culture of Sf9 Cells and Preparation of Microsomes. Sf9 insect cells were cultured under the conditions in our previous study with slight modifications (Ishii et al., 2014). In short, the cells were grown in a $500 \mathrm{ml}$ plastic Erlenmeyer flask (Corning, Lowell, MA) containing Sf-900II medium (Life Technologies) supplemented with $5 \% \mathrm{FBS}$ and $10 \mu \mathrm{g} / \mathrm{ml}$ gentamycin. To obtain recombinant baculovirus,

ABBREVIATIONS: BSA, bovine serum albumin; CNX, calnexin; COPI, coat protein I complex; CT, cytosolic tail; DAPI, 4',6-diamidino-2phenylindole; DM, dilysine motif; ER, endoplasmic reticulum; HA, hemagglutinin; HA-tag, hemagglutinin epitope-tag; HLM, human liver microsomes; HRP, horseradish peroxidase; PCR, polymerase chain reaction; TM, transmembrane; UGT, UDP-glucuronosyltransferase; WT, wild-type. 
Sf9 cells $\left(2 \times 10^{7}\right.$ cell) were seeded in a $175 \mathrm{~cm}^{2}$ t-flask (Thermo Fisher Scientific) and then transfected with recombinant bacmids with CellfectinII reagent (Life Technologies) diluted with Sf-900II medium in the absence of FBS or antibiotics. After 5 hours of incubation, the medium was replaced with fresh medium, and the cells were cultured for 7 days. The cells were pelleted by low-speed centrifugation, and the supernatant was collected as primary (P1) virus. The pelleted cells were resuspended in $10 \mathrm{ml}$ of PBS, and an aliquot of the suspension was mixed with the same volume of $2 \times$ SDS-PAGE sampling buffer [125 mM Tris-HCl (pH 6.8); 4\% SDS; 20\% glycerol; 0.006\% bromophenol blue; $10 \%$ 2-mercaptoethanol] to prepare whole-cell lysate for confirmation of target protein expression. Titer of the recombinant baculovirus was determined using a BacPAK qPCR Titration Kit (Clontech, Mountain View, CA). Baculoviral titer was amplified by several rounds of transfection until a titer of over $1.0 \times 10^{7}$ plaque-forming units $/ \mathrm{ml}$ was obtained. For the expression of recombinant enzymes, Sf9 cells $\left(2 \times 10^{6}\right.$ cells $\left./ \mathrm{ml}, 200 \mathrm{ml}\right)$ were infected with recombinant baculovirus, and cultured for 48 hours. Microsomes were prepared from them according to the protocols described previously (Ishii et al., 2014).

Construction of Recombinant Mammalian Expression Vectors. cDNA of CD4 was purchased from Ori-Gene Technologies (Rockville, MD) and amplified by PCR with a pair of primers: sense, $5^{\prime}$ CCGCTCGAGACAATGAACCGGGGAGTCCCTTTTAGG-3' (sense primer A; underlining indicates XhoI site); anti-sense, 5'-CGGGGTACCTCAAATGGGGCTACATGTCTTC-3' (underlining indicates KpnI site). The PCR product was digested with XhoI and KpnI and subcloned into the pcDNA3.1/hygro(-) vector for transient expression in COS-1 cells. A deletion mutant of CD4 lacking TM and cytoplasmic domains (CD4 $\Delta \mathrm{TM})$ was generated by QuickChange site-directed mutagenesis with a pair of primers: sense, 5' -CCACCCCGGTGCAGCCATGAGGTACCAAG-3'; anti-sense, 5' -CTTGGTACCTCATGGCTGCACCGGGGTGG-3'. The mutagenesis was conducted with the pcDNA3.1/hygro(-) CD4 construct as a template under the following thermal cycle conditions: initial denaturation, $94^{\circ} \mathrm{C}, 2$ minutes; cycling step $\left(\times 25\right.$ rounds): $98^{\circ} \mathrm{C}$, 10 seconds; $52^{\circ} \mathrm{C}, 30$ seconds; $68^{\circ} \mathrm{C}, 4$ minutes; extra extension, $68^{\circ} \mathrm{C}$, 5 minutes; hold, $4^{\circ} \mathrm{C}$. The PCR product was digested with DpnI, and used for transformation. A chimeric protein of CD4 (CD4-UGT), which replaced the cytoplasmic domain of CD4 with that of UGT2B7 was generated by sequential PCRs. Each PCR product was used as a template in the next round of PCR. Pairs of primers used in each round of PCR are listed below: first round sense, sense primer A; first round anti-sense, $5^{\prime}$-GAAACAAAACAGACAACATTTGAAGAAGATGCCTAGCCC-3' (bold, sequence coding the end of TM of CD4); second round sense, 5'-CCGCTCGAGACAATGAACC-3' (sense primer $\mathrm{B}$, underlining indicates XhoI site); second round anti-sense, 5' - TTCTTTGCTTTTCTAGCAAACTTCCAGAAACAAAACAGACAACATTTG-3'; third round sense, sense primer B; third round anti-sense, $5^{\prime}$-GGGGTACCCTAATCATTTTTTCCCTTCTTTGCTTTTCTAGCAAAC-3' (underlining indicates KpnI site). Products of the third round PCR were subcloned into the pcDNA3.1/hygro(-) vector using XhoI-KpnI sites. The addition of an HA-tag to the C-terminus and alanine mutation of the DM of CD4-UGT were conducted by sitedirected mutagenesis with pairs of primers: sense for addition of the HA-tag, 5' -GCTAGAAAAGCAAAGAAGGGAAAAAATGATTACCCATACGATGTTCCAGATTACGCTTAGGGTACCAAGCTTA-3' (underlining indicates HA-tag); anti-sense for addition of the HA-tag, 5' TAAGCTTGGTACCCTAAGCGTAATCTGGAACATCGTATGGGTAATCATTTTTTCCCTTCTTTGCTTTTCTAGC-3' (underlinining indicates HA-tag); sense for alanine mutation of the DM, 5'-GTTTCTGGAAGTTTGCTAGAAAAGCAAAGGCGGGAGCAAATGATTAGGGTACCAAGCTTAAGTTTA-3' (underlining alanine mutations); anti-sense for alanine mutation of the DM, 5' -TAAACTTAAGCTTGGTACCCTAATCATTTGCTCCCGCCTTTGCTTTTCTAGCAAACTTCCAGAAAC-3' (underlining alanine mutations). Mutagenesis was conducted with pcDNA3.1/hygro(-)-CD4-UGT as template in a two-step cycle. PCR products were treated with DpnI and used for transformation to obtain the desired constructs. DNA of UGT2B7 WT and WT-HA were subcloned from pFastBac1 to pcDNA3.1/hygro(-) vector using
KpnI-sites. The HA-tag was also added to the C-terminal end of $\Delta \mathrm{DM}, \Delta \mathrm{CT}$, and $\Delta \mathrm{TM}$ by site-directed mutagenesis using pFastBac1 constructs as templates. Primers used in the mutagenesis are listed in Supplemental Table 2, and amplification was carried out in a two-step cycle, as mentioned above. After HA-tag addition, cDNAs of $\triangle \mathrm{DM}-\mathrm{HA}, \triangle \mathrm{CT}-\mathrm{HA}$, and $\triangle \mathrm{TM}-\mathrm{HA}$ were subcloned into pcDNA3.1/hygro(-) as was done with WT-HA. In addition, mammalian constructs coding UGT2B7 $\Delta 1-\Delta 11$ with HA-tag were generated by twostep cycle mutagenesis. Primers and templates used for preparing $\Delta 1-\Delta 5$ and $\Delta 6-\Delta 11$ are listed in Supplemental Tables 3 and 4 , respectively. After DpnI treatment, reaction mixtures were used for the transformation of $E$. coli. Nucleotide sequences were checked according to the protocol described above.

Culture of COS-1 Cells and Transfection of Mammalian Expression Vector. COS-1 cells were grown in Dulbecco's modified Eagle medium containing high glucose (Wako Pure Chemical, Osaka, Japan) and $10 \%$ FBS. For immunoblotting, cells were seeded in 35-mm dishes the day before transfection, and pcDNA3.1 vectors $(2 \mu \mathrm{g})$ were transfected with FuGENE6 (Promega, Madison, WI) according to the manufacturer protocol. Twenty-four hours after transfection, the cells were lysed in lysis buffer, $20 \mathrm{mM}$ Tris-HCl ( $\mathrm{pH} 7.4$ ) containing $150 \mathrm{mM} \mathrm{NaCl}, 1 \%$ Triton X-100, 10\% glycerol, and protease inhibitor cocktail (Nacalai Tesque, Kyoto, Japan); and sonicated to prepare whole-cell lysates. For immunofluorescence staining, cells were plated onto 13-mm coverslips the day before transfection. The amount of transfected pcDNA3.1 was $1 \mu \mathrm{g}$ with FuGENE6 as a transfection reagent, and immunofluorescence staining was performed 24 hours after transfection.

Immunoblotting. Proteins separated by SDS-PAGE were electroblotted onto a polyvinylidene difluoride membrane (Merck Millipore, Burlington, MA). UGT2B7 was detected either by goat anti-mouse low pI form UGT antibody (Mackenzie et al., 1984) or rabbit anti-HA-tag antibody (Sigma-Aldrich, St. Louis, MO). Rabbit anti-CNX (GeneTex, Irvine, CA) and mouse anti- $\beta$-actin (Sigma-Aldrich) were purchased from the sources indicated. The primary antibodies were diluted 2000 -fold when used. Immunochemical detection was conducted either with horseradish peroxidase (HRP)-conjugated secondary antibodies, HRP-rabbit anti-goat IgG (MP Biomedicals, Santa Ana, CA) or HRP-donkey anti-rabbit IgG (GE Healthcare, Little Chalfont, UK). The secondary antibodies were diluted 10,000-fold in use. EzWestLumi plus (ATTO, Tokyo, Japan) was used as a substrate of HRP, and the signals were visualized with a ChemiDoc MP System (Bio-Rad, Hercules, CA) and quantified with ImageJ software.

Immunofluorescence Microscopy. Immunofluorescence staining was performed according to the protocol described previously with slight modification (Hirota and Tanaka, 2009; Fujimoto et al., 2015). Briefly, COS- 1 cells were immobilized on coverslips with $4 \%$ paraformaldehyde in PBS ( $\mathrm{pH}$ 7.4) for 30 minutes at room temperature, and sequentially quenched with $50 \mathrm{mM} \mathrm{NH}_{4} \mathrm{Cl}$ in $\mathrm{PBS}$ for 15 minutes. Permeabilization and blocking was simultaneously conducted by treating the samples with PBS containing $0.05 \%$ saponin and $1 \%$ bovine serum albumin (BSA; fraction V) for 30 minutes. The cells were incubated with the following primary antibodies for 1 hour at room temperature: rabbit anti-CD4 antibody (H-370; Santa Cruz Biotechnology, Dallas, TX); rabbit anti-HA-tag antibody (Sigma-Aldrich); rabbit anti-UGT2B7 antibody (Proteintech, Rosemont, IL); and mouse anti-CNX antibody (BD Biosciences, San Jose, CA). The cells were diluted 300 -fold with PBS containing $0.05 \%$ saponin and $1 \%$ BSA. After a PBS wash $(5$ minutes, $\times 3$ ), the cells were incubated for 30 minutes at room temperature with Alexa Fluor 488- and Cy3labeled secondary antibodies, together with 4',6-diamidino-2-phenylindole (DAPI) as a nuclear counterstain. The secondary antibody was diluted 300 -fold with PBS containing $0.05 \%$ saponin and $1 \%$ BSA, and the concentration of DAPI was $1 \mu \mathrm{g} / \mathrm{ml}$ in use. After a PBS wash (5 minutes, $\times 3$ ), coverslips were mounted in MOWIOL 4-88 (Merck Millipore) onto glass slides, and the samples were analyzed by the LSM 700 Laser-Scanning Confocal Microscope (Carl Zeiss, Oberkochen, Germany) equipped with four lasers $(405,455,555$, and $639 \mathrm{~nm})$. 
The objective lens used in the analysis was Plan-Apochromat $63 \times / 1.4$ oil differential interference contrast. To obtain multiple stained images, laser and filter were optimized automatically for each fluorescence label. Image files were created from raw data with ZEISS Efficient Navigation (ZEN) software, and photographic images were processed using Photoshop (Adobe Systems, San Jose, CA). Colocalization of UGT2B7 and CNX were quantified with ZEN software.

Detection of Aggregated Forms of UGT2B7-HA and Its Mutants by Triton X-100 Treatment. Triton X-100 soluble and insoluble fractions were prepared as described previously with slight modifications (Hirota and Tanaka, 2009). In brief, COS-1 cells transiently expressing UGT2B7-HA and its mutants were harvested in cold sucrose solution ( $0.25 \mathrm{M}$ sucrose, $1 \mathrm{mM}$ EDTA, $10 \mathrm{mM}$ Hepes, $\mathrm{pH}$ 7.4), homogenized by 25 passages through a 23 -gauge needle connected to a 1-ml syringe, and subsequently centrifuged at $1000 \mathrm{rpm}$ for 10 minutes. The resulting postnuclear supernatants (homogenate) were centrifuged at 105,000g for 1 hour, and fractionated into membrane (pellet) and cytosol (supernatant). Membrane fractions were solubilized in $1 \%$ Triton X-100 in PBS, and divided into soluble and insoluble fractions by centrifugation $\left(105,000 \mathrm{~g}, 4^{\circ} \mathrm{C}, 1\right.$ hour $)$. Insoluble fractions were further solubilized in radioimmunoprecipitation assay buffer, as follows: $50 \mathrm{mM}$ Tris- $\mathrm{HCl}$ (pH 7.5) containing $150 \mathrm{mM} \mathrm{NaCl}, 1 \mathrm{mM}$ EDTA, $1 \% \mathrm{NP} 40,0.1 \%$ SDS, and $0.05 \%$ sodium deoxycholate. Volumes of each fraction were kept equal, and then homogenate ( $15 \mu \mathrm{g}$ protein) and equivalent volumes of the later fractions were analyzed by immunoblotting.

Treatments with Proteasome and Lysosome Inhibitors. UGT2B7-HA, $\triangle$ CT-HA, and $\Delta$ TM-HA were transiently expressed in COS-1 cells. Twelve hours after transfection, the cells were treated with $1 \mu \mathrm{M}$ proteasome inhibitor (MG132) and lysosome inhibitor cocktail, $10 \mu \mathrm{g} / \mathrm{ml} \mathrm{E}-64 \mathrm{~d}, 20 \mu \mathrm{g} / \mathrm{ml}$ leupeptin, and $10 \mu \mathrm{g} / \mathrm{ml}$ pepstatin A for 12 hours. Whole-cell lysates were prepared and analyzed by immunoblotting.

A

23

WT

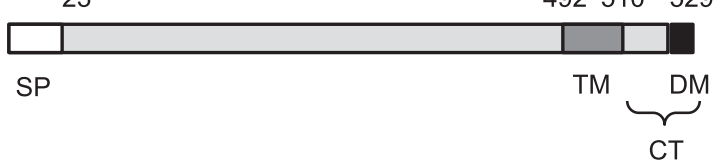

$\triangle \mathrm{DM}$

$(\Delta 525-529)$

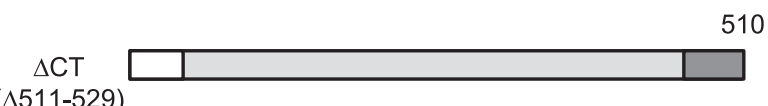

$(\Delta 511-529)$

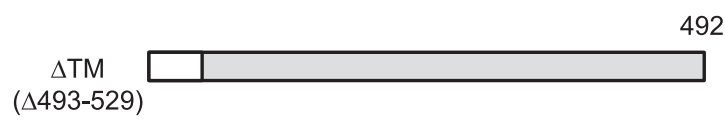

B

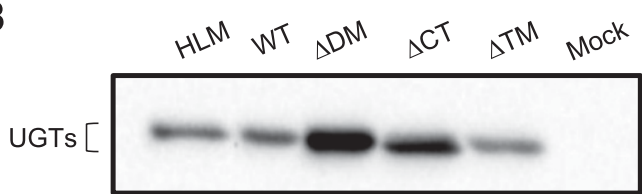

Secretion of the Cytoplasmic and Transmembrane DomainDeleted Mutants of UGT2B7 and CD4. COS-1 cells were transfected with each construct and cultured for 12 hours in $1 \mathrm{ml}$ of FBS-free Dulbecco's modified Eagle medium. Cells were harvested in cold sucrose solution, and whole lysates were prepared as described above. Medium was also collected and $400 \mu \mathrm{l}$ of medium was mixed with $100 \mu \mathrm{l}$ of $50 \%$ trichloroacetic acid, and incubated on ice for 10 minutes. After centrifugation, precipitants were washed with ice-cold acetone twice and analyzed by immunoblotting.

Other Methods. Protein concentrations of baculosomes were determined by the method of Lowry et al. (1951), and those of COS-1 cell lysates were by Protein Assay CBB Solution (Nacalai Tesque) with BSA as a standard.

\section{Results}

To assess the role of the C-terminal region of UGT2B7, including the DM, in its ER localization, we designed a series of deletion mutants lacking the $\Delta \mathrm{DM}, \Delta \mathrm{CT}$, and $\Delta \mathrm{TM}$, respectively, as illustrated in Fig. 2A. Each deletion mutant, as well as UGT2B7 WT, were expressed in Sf9 insect cells, and their expression was analyzed by immunoblotting with goat anti-mouse low pI form UGT antibody (Mackenzie et al., 1984). Using this antibody, we observed a single band in cell lysates expressing each UGT2B7 construct, which had the predicted molecular weight based on the size of the deletion (Fig. 2B). To determine subcellular localization of the UGT2B7 mutants, we fractionated the homogenates prepared from insect cells simultaneously infected with recombinant baculovirus coding human CNX as an ER marker. We obtained three fractions,
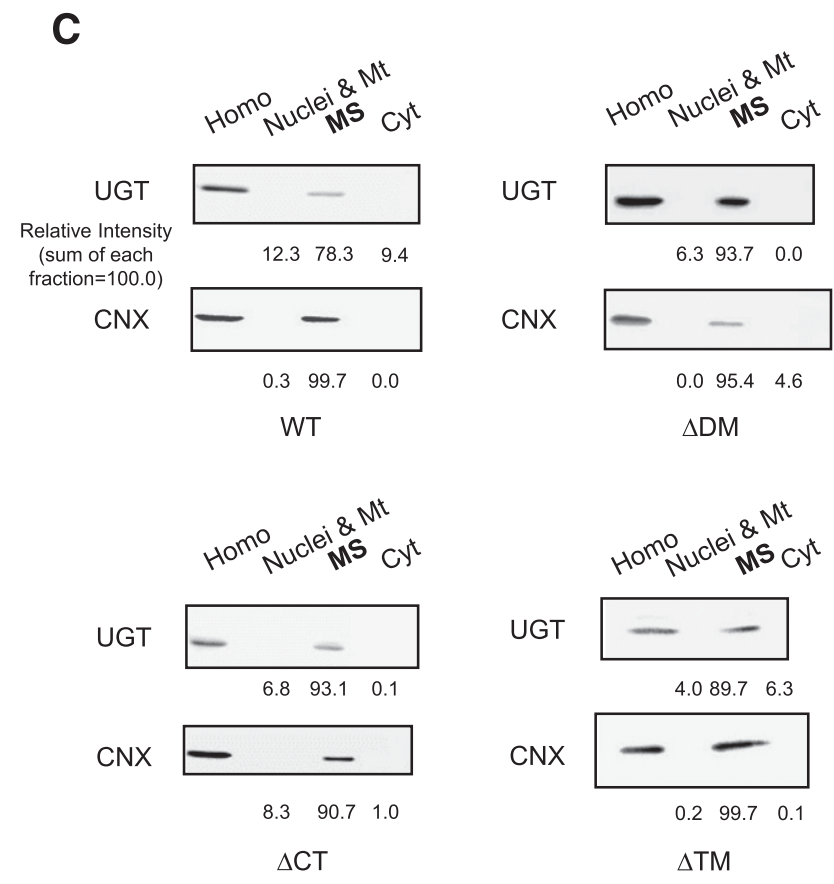

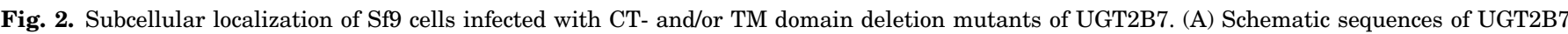

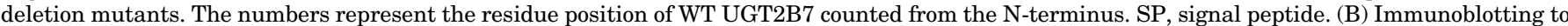

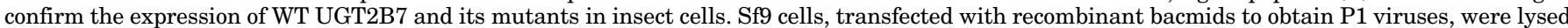

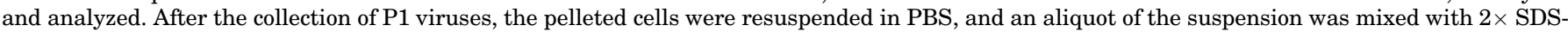

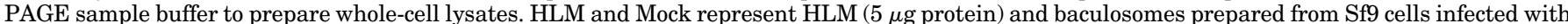

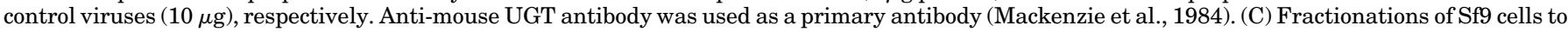

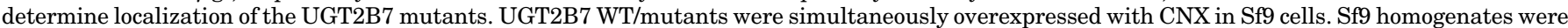

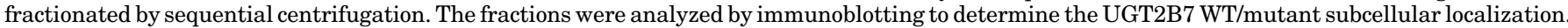

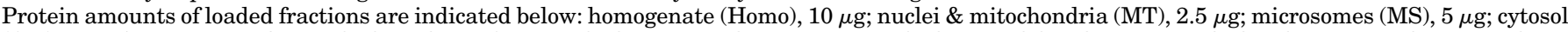

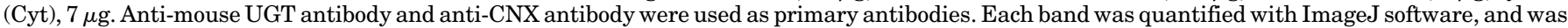
shown as the percentage of the total. 
A

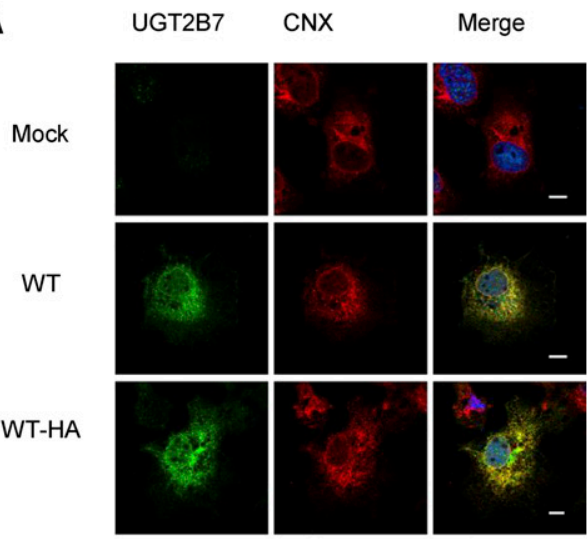

B

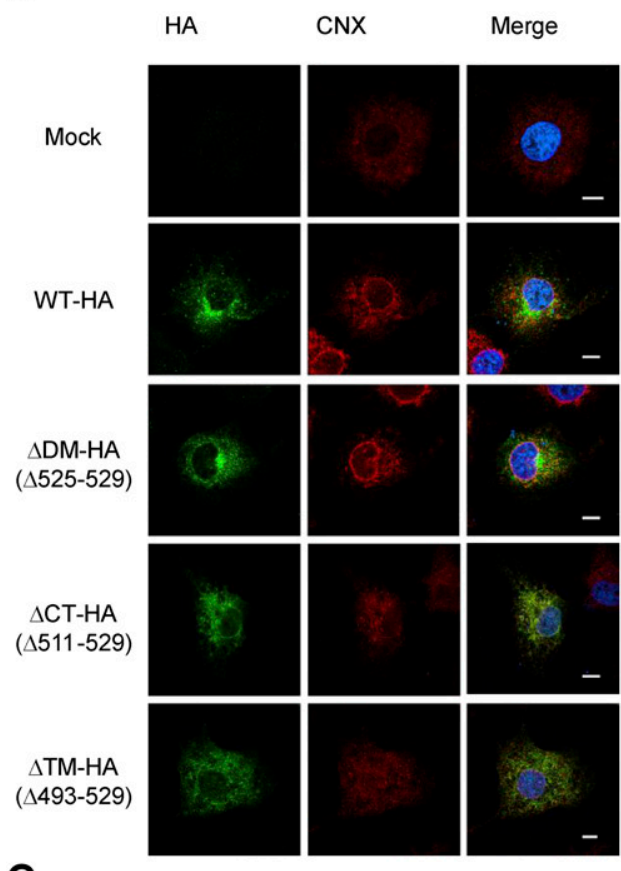

C

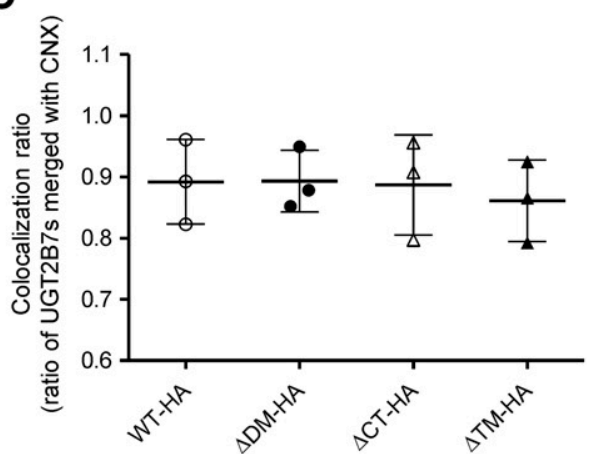

D CD4

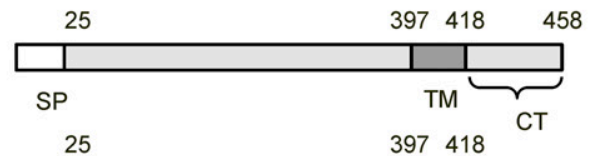

CD4-UGT

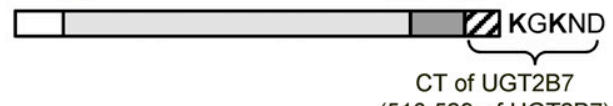

(510-529 of UGT2B7)
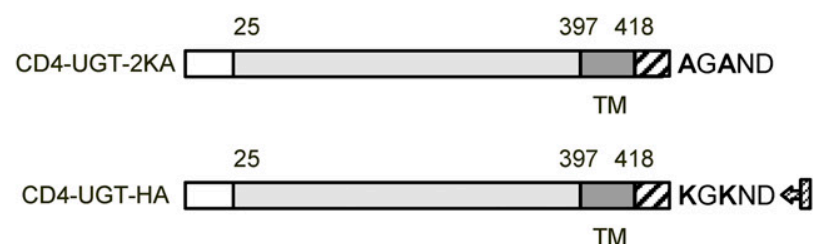

\& 6 : HA-tag

$\mathbf{E}$

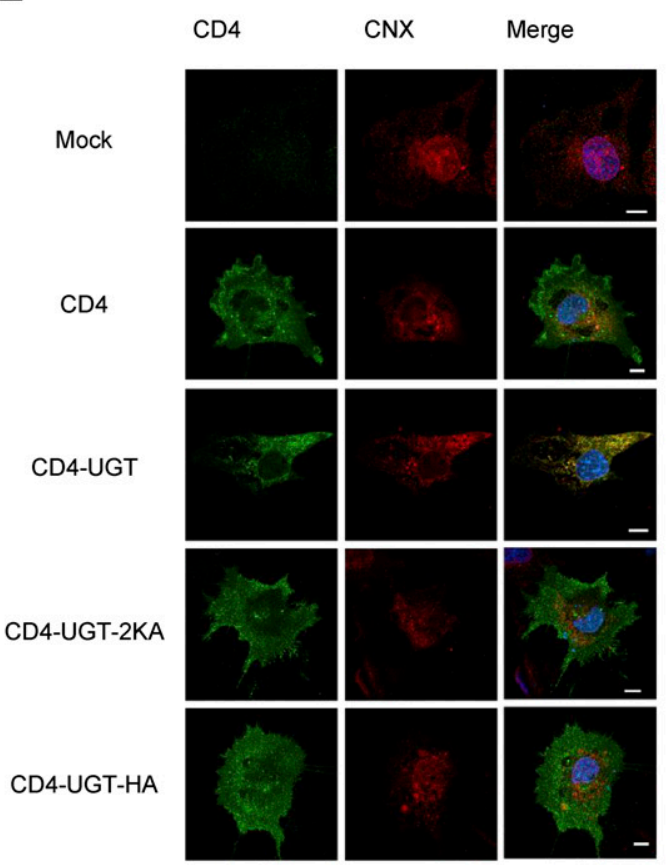

Fig. 3. Localization of the CT and/or TM domain-deleted mutants of UGT2B7 and the CD4-UGT chimeric protein in COS-1 cells. COS-1 cells transfected with UGT2B7 WT or the mutants were fixed and stained 24 hours after transfection. (A) Localization of UGT2B7 and UGT2B7-HA (UGT2B7 with a C-terminal HA-tag) were compared with anti-UGT2B7 antibody (green). Cells were also stained red with an antibody to endogenous CNX, a marker protein of the ER. In the merged panels, nuclei stained by DAPI are shown in cyan. (B) Localization of the C-terminal-deleted mutants of UGT2B7. The sequences of the mutants are shown in Fig. 2A. An HA-tag was conjugated to their C-terminus for immunochemical detection. Cells were stained with anti-HA antibody (green) and anti-CNX (red). (C) Colocalization of UGT2B7 WT/mutants and CNX were quantified, and the mean \pm S.D. values are shown. (D) Schematic sequences of CD4 and its chimeric proteins whose cytoplasmic domains were replaced with that of UGT2B7. The numbers represent the residue position of WT CD4 counted from the N-terminus. The DM of UGT2B7 is highlighted in the C-terminus of each chimera. SP, signal peptide. (E) Localization of CD4 chimeric proteins. CD4-UGT represents a CD4 chimera whose cytoplasmic domain was replaced with that of UGT2B7. Cells were stained with anti-CD4 antibody, which recognizes the extracellular domain of CD4 (green) and anti-CNX (red). In merged panels, the nuclei are shown in cyan. Scale bar, $10 \mu \mathrm{m}$. 
nuclei and mitochondria, microsomes, and cytosol, for each sample. All of the deletion mutants were exclusively collected in the microsomal fraction, similar to CNX (Fig. 2C), in agreement with our last report (Miyauchi et al., 2015). To further investigate their subcellular localization, we conducted immunofluorescence staining to analyze colocalization of UGT2B7 and endogenous CNX in COS-1 cells. Overexpressed WT UGT2B7 showed a reticular staining pattern and colocalized with CNX (Fig. 3A, top and middle panels). Unfortunately, the epitope of UGT2B7 recognized by the anti-UGT2B7 antibody is not clear. Accordingly, in later analyses using several deletion mutants, we added an HA-tag to the C-terminus to detect the mutants using an immunochemical approach. Before detection of the mutant with the anti-HA-tag antibody, we determined whether the HA-tag affects the localization of UGT2B7 WT. As shown in Fig. 3A, both HA-tagged and non-tagged UGT2B7 WTs were recognized equally by anti-UGT2B7 antibody, and the addition of the HA-tag to the C-terminal end did not alter the staining pattern. Next, we conducted staining of the C-terminal deletion mutants with anti-HA-tag antibody. As shown in Fig. $3 \mathrm{~B}, \Delta \mathrm{DM}, \Delta \mathrm{CT}$, and $\Delta \mathrm{TM}$ mutants colocalized with CNX, similar to WT-HA, indicating that about $80 \%-95 \%$ of UGT2B7 WT/deletion mutants were colocalized with CNX (Fig. 3C). These results support the results of the fractionation approach (Fig. 2C). A chimeric protein that replaced the cytoplasmic domain of CD4 with that of UGT2B7 (CD4-UGT) also colocalized with CNX. However, it was redistributed to the cell surface by the substitution of two lysines with alanines within the cytoplasmic domain (CD4-UGT-2KA) (Fig. 3, D and E), suggesting that the DM acts as an ER retention signal in this chimera. Intriguingly, in contrast to the case of UGT2B7 (Fig. 3A), fusion of the HA-tag to the C-terminus of CD4-UGT (CD4-UGT-HA) altered the ER distribution of CD4-UGT to the cell surface (Fig. 3, D and E), consistent with a previous report
A
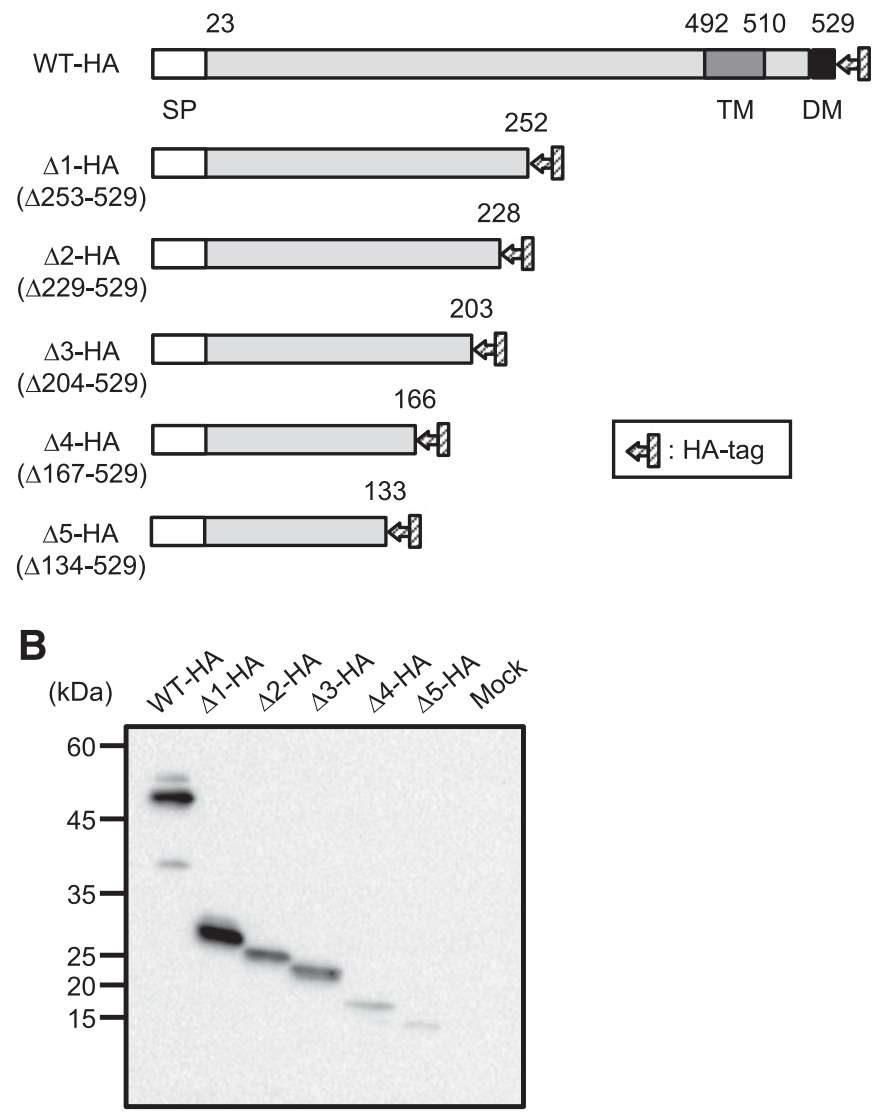

C

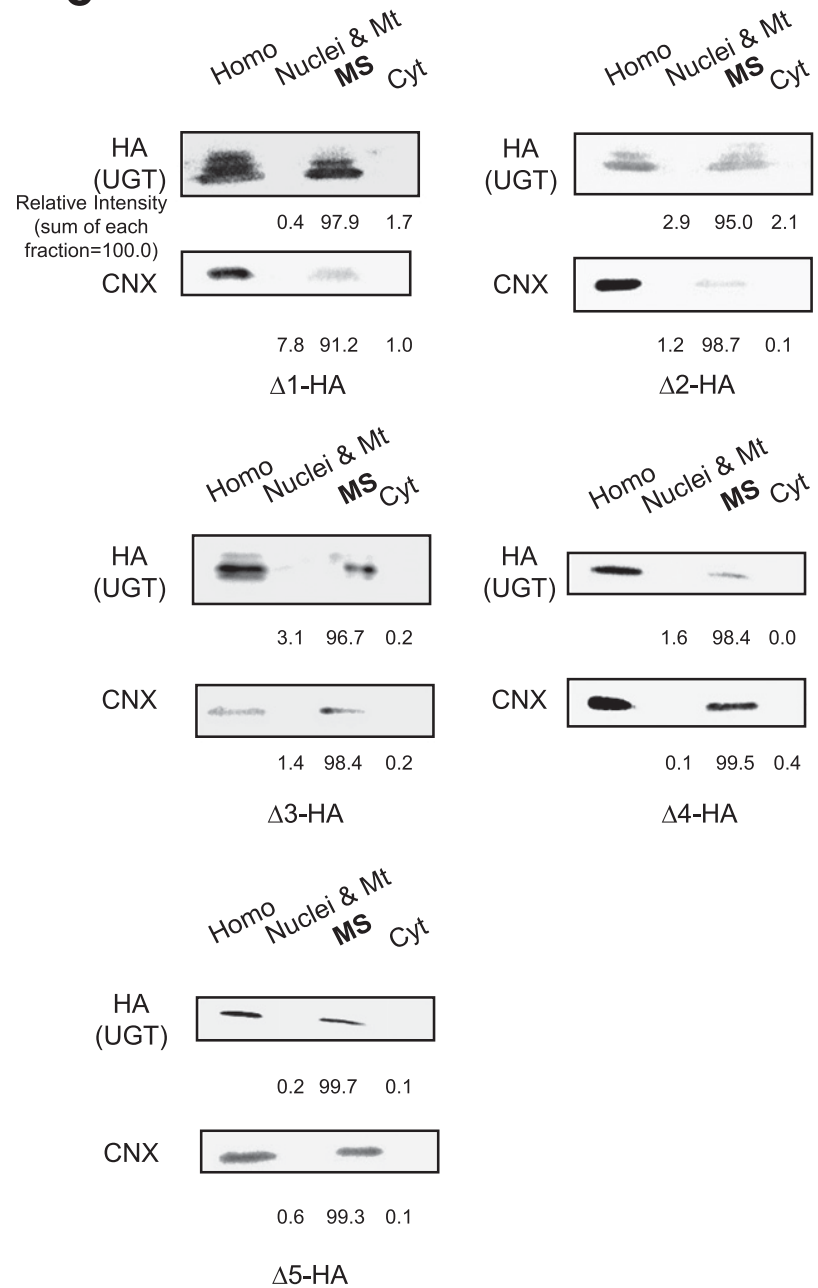

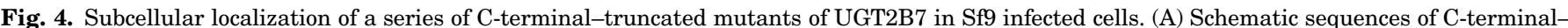

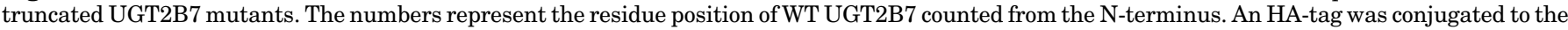

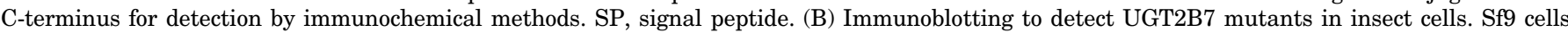

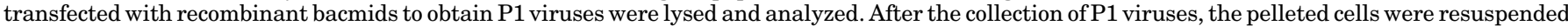

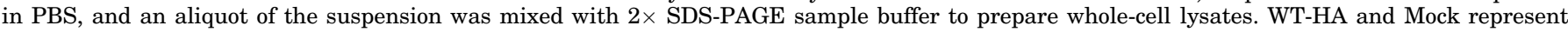

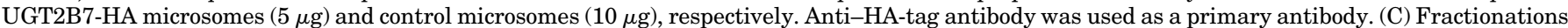

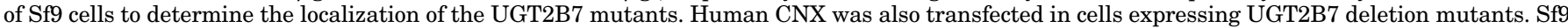

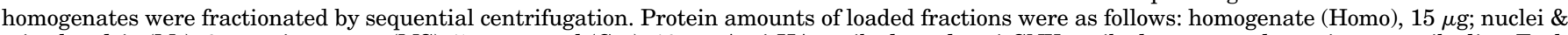

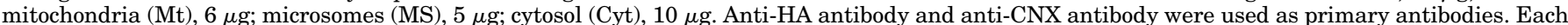
band was quantified with ImageJ software and was shown as a percentage of the total. 
demonstrating that the proper positioning of two lysine residues from the C-terminus is critical for the retention of ER resident membrane proteins (Jackson et al., 1990). Collectively, our data suggest that the DM within the cytoplasmic domain of UGT2B7 is sufficient to localize CD4 to the ER but is not necessary for the ER retention of UGT2B7.

The results described above led us to speculate that the luminal domain of $\mathrm{UGT} 2 \mathrm{~B} 7$ is required for ER retention. In addition, it has been reported that the 141-240 region of human UGT1A6 is another membrane-associated domain and functions as an ER retention signal (Ouzzine et al., 1999). When this region was transplanted to the cytoplasmic enhanced GFP, the UGT 141-240/enhanced GFP chimera was localized to the ER. Based on these findings, we first focused on the N-terminal half-domain of UGT2B7 (24-252; except signal peptide) to explore the regions necessary for ER retention. We generated five deletion mutants $(\Delta 1-\mathrm{HA}$ to $\Delta 5$-HA) systematically lacking 30 residue sections containing two or three predicted helices from the C-terminal end of UGT2B7 (24-252), and an HA-tag was added to the C-terminal end of each deletion mutant (Fig. 4A). We transiently expressed these UGT2B7 mutants in Sf9 insect cells and confirmed their expression by immunoblotting (Fig. 4B). We further performed subcellular fractionation of insect cells expressing these deletion mutants using the same procedure, as in Fig. 2C. The results showed that all of the mutants were exclusively detected in the microsomal fraction with coexpressed CNX (Fig. 4C). Moreover, consistent with the results of subcellular fractionation, the results of immunofluorescence microscopy in COS-1 cells also showed that all of the deletion mutants colocalized with CNX in reticular-like structures (Fig. 5A), and that $90 \%$ of these mutants colocalized with CNX (Fig. 5B). Taken together, these results suggest that at least the N-terminal 24-133 region may be necessary for the retention of UGT2B7 in the ER.

To verify the necessity of the 24-133 residue region for ER retention of UGT2B7, we created a deletion mutant of this domain ( $\triangle 6$-HA) and analyzed its expression and localization in COS-1 cells (Fig. 6, A-C). To our surprise, UGT2B7 $\Delta 6$-HA localized to CNX-positive reticular-like structures. We further generated a series of deletion mutants of the UGT2B7 luminal domain (Fig. 6A). These deletion mutants were transiently expressed in COS-1 cells, and their expression and localization analyzed using immunoblotting and immunofluorescence microscopy, respectively (Fig. 6, B and C). As shown in Fig. 6C, despite the systematic truncation, all of the mutants were observed in the reticular-like structures and colocalized with CNX. Moreover, $80 \%-95 \%$ of the serial mutants colocalized with CNX (Fig. 6D) as same as UGT2B7 WT (Fig. 3C). Collectively, these data imply that UGT2B7 could localize in the ER without any signal sequence rather than with the presence of a possible signal for the retention to the ER. Moreover, it is well known that proteins that aggregate in cells are largely detergent insoluble (Imai et al., 2001; Hirota and Tanaka, 2009). When we solubilized microsomes expressing these deletion mutants with $1 \%$ Triton $\mathrm{X}-100$, they were predominantly recovered in solubilized fractions (Fig. 7A), indicating that they were properly folded, and thereby ruling out the possibility that the ER localization of these mutants resulted from accumulation in the ER by unfolding or misfolding. Moreover, we examined the stability of UGT2B7-HA deletion mutants ( $\Delta \mathrm{CT}$ and $\Delta \mathrm{TM})$ using inhibitors of proteasome

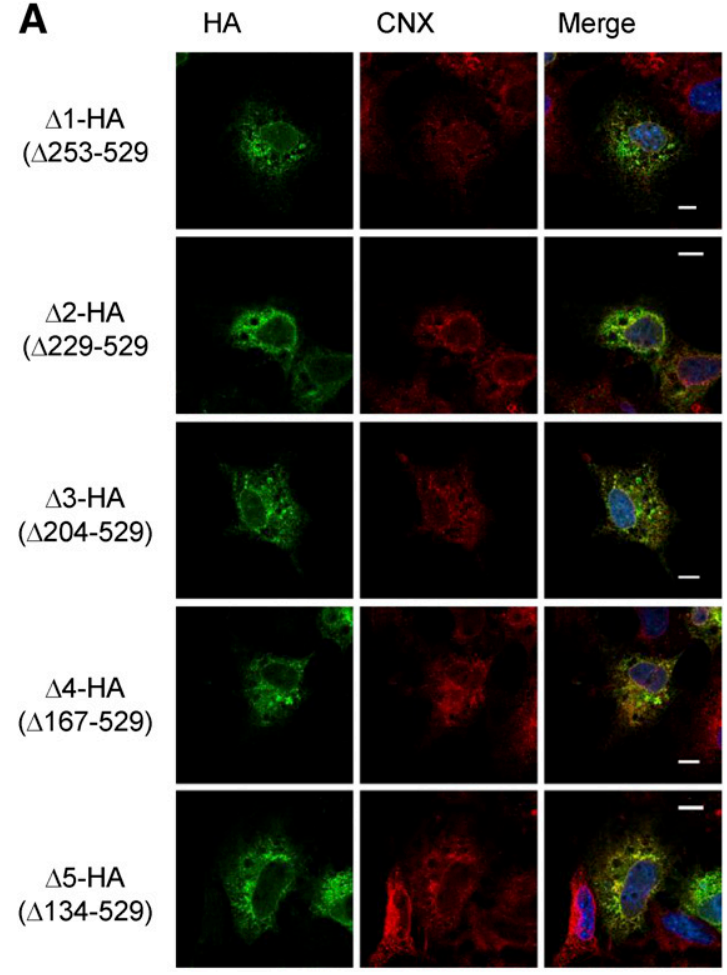

B

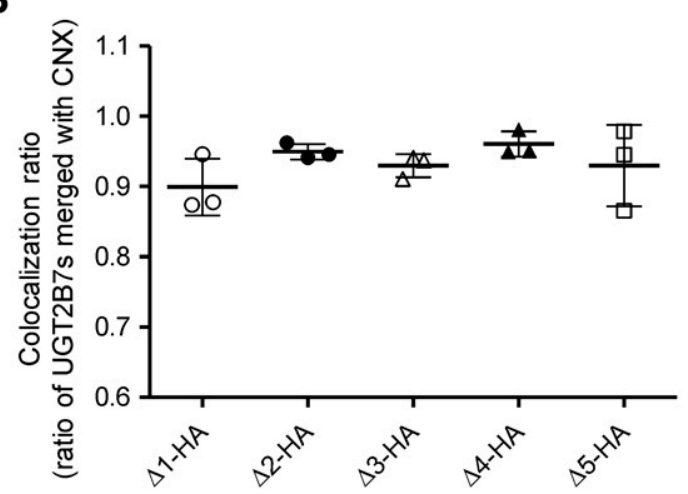

Fig. 5. (A) Localization of C-terminal-truncated mutants of UGT2B7 in transfected COS-1 cells. Schematic sequences of the mutants are presented in Fig. 4A. All of the mutants were fused with an HA-tag at their C-termini for immunochemical detection. COS-1 cells transiently expressing the mutants were stained with anti-HA antibody (green). Endogenous CNX was also visualized with anti-CNX antibody (red) as an ER marker. Nuclei stained with DAPI are shown in cyan. Scale bar, $10 \mu \mathrm{m}$ in the merge panels. (B) Colocalization of UGT2B7 mutants and CNX were quantified, and the mean \pm S.D. values are shown.

and lysosomal degradation. Treatment with MG132, a proteasome inhibitor, but not with lysosomal protease inhibitors, increased protein levels of WT, $\Delta \mathrm{CT}$, and $\Delta \mathrm{TM}$ of UGT2B7 (Fig. 7, B and C), thereby suggesting that the deletion of both the cytoplasmic and TM domains did not affect the stability of mutant proteins.

Deletions of the TM domain of membrane proteins transported by the secretory pathway should result in their secretion into the medium, unless they contain a retention signal in the remaining domain (Pääbo et al., 1986, 1987). We examined whether both cytoplasmic- and TM domain-deleted mutants of UGT2B7 and CD4 were secreted. Although all of the deletion mutants of UGT2B7-HA ( $\Delta \mathrm{TM}, \Delta 1$, and $\Delta 7)$ were 
A

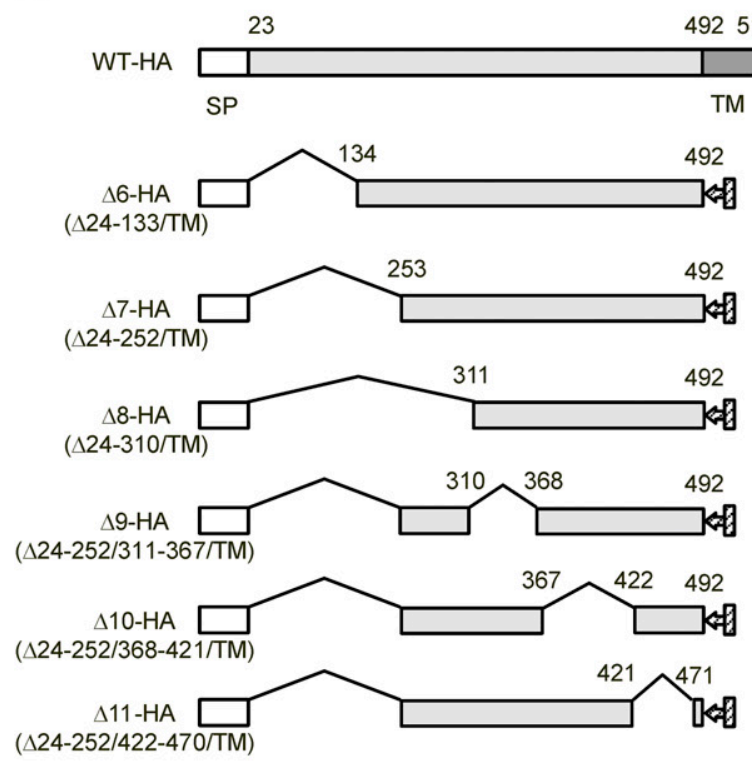

B

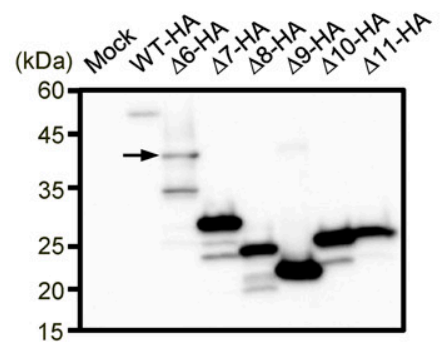

D

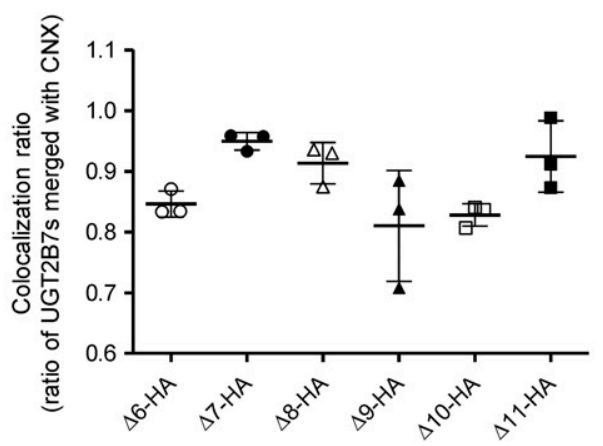

C
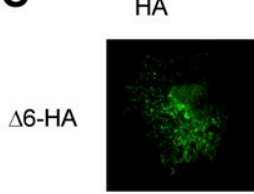

CNX

Merge
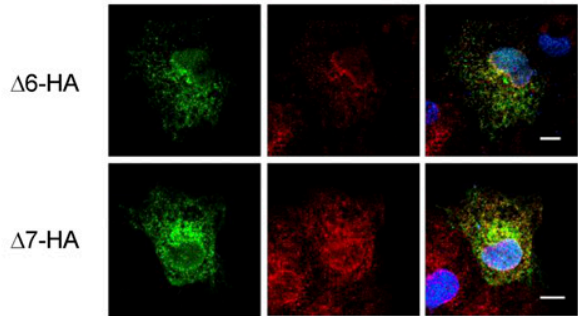

$\triangle 8-H A$
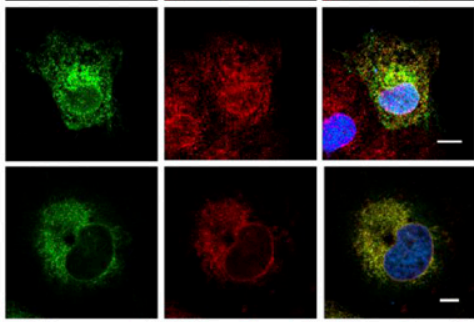

$\triangle 9-H A$
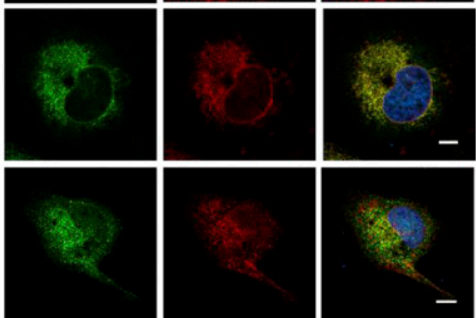

$\triangle 10-\mathrm{HA}$
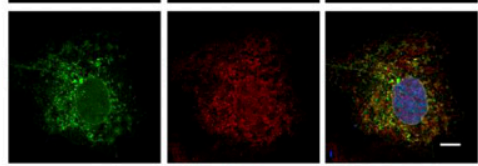

$\Delta 11-\mathrm{HA}$
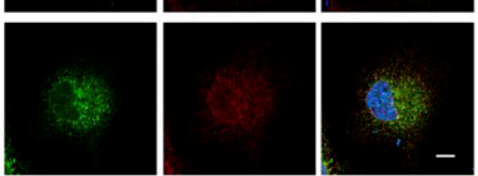

Fig. 6. Localization of internal deletion mutants of UGT2B7 in transfected COS-1 cells. (A) Schematic sequences of UGT2B7 mutants ( $\Delta 6$-HA to $\Delta 11-H A)$. (B) Immunoblotting to detect the UGT2B7 mutants in COS-1 cells. Cells were transfected with $2 \mu \mathrm{g}$ of vector coding each UGT2B7 mutant and lysed 24 hours after transfection. The lysates $(20 \mu \mathrm{g})$ were analyzed by immunoblotting. Anti-HA antibody was used as a primary antibody. (C) Immunofluorescence to show colocalizations of the UGT2B7 mutants with CNX. The mutants were transiently expressed in COS-1 cells and visualized with anti-HA antibody (green). Endogenous CNX was also stained with anti-CNX antibody (red) as an ER marker. Nuclei were stained with DAPI and are shown in cyan. Scale bar, $10 \mu \mathrm{m}$ in the merge panel. (D) Colocalization of UGT2B7 mutants and CNX were quantified, and the mean \pm S.D. values are shown.

detected only in cell lysates, CD4- $\Delta$ TM was significantly secreted into the cultured medium (Fig. 7D).

\section{Discussion}

The DM is one representative peptide for motif-based retrograde transportation, but it is controversial whether this motif is indeed necessary for ER localization of UGTs. In this study, we present evidence that C-terminal deletion mutants of UGT2B7, including $\triangle \mathrm{DM}, \Delta \mathrm{CT}$, and $\Delta \mathrm{TM}$, can localize to the
ER in their folded states (Fig. 2C; Fig. 3, B and C; Fig. 7). It has been demonstrated previously that the proper positioning of two lysines at -3 and -4 or -5 from the $\mathrm{C}$-terminus is critical for the retention of ER resident membrane proteins, because the addition or deletion of residues from the C-terminus decreased retention efficiency (Jackson et al., 1990). Indeed, we also confirmed this finding, using the chimeric protein CD4-UGT (Fig. 3, D and E). However, our findings that not only UGT2B7ADM, but also UGT2B7-HA, in which the positions of two lysine residues were shifted from -3 and -5 to -12 
A

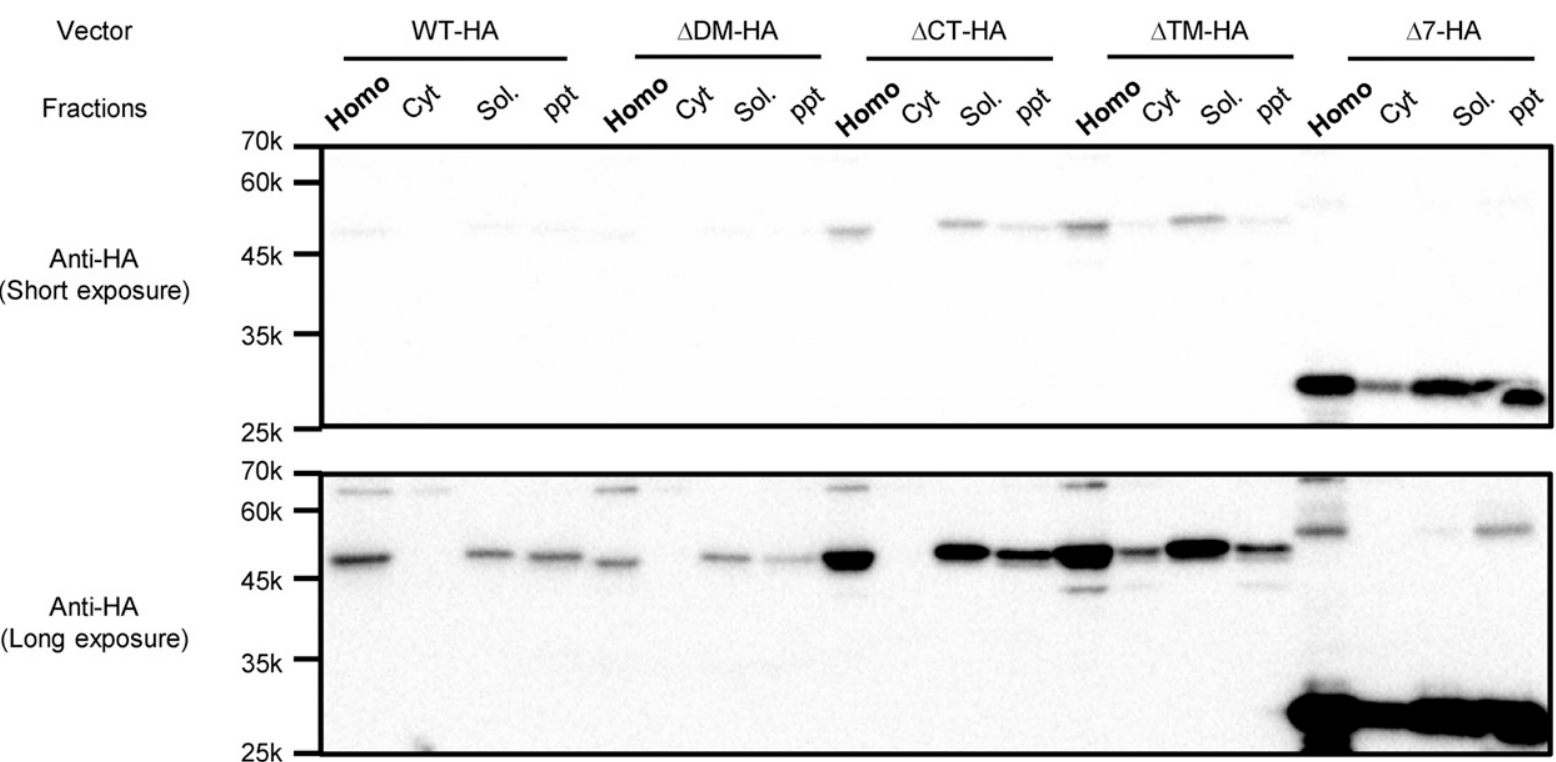

B

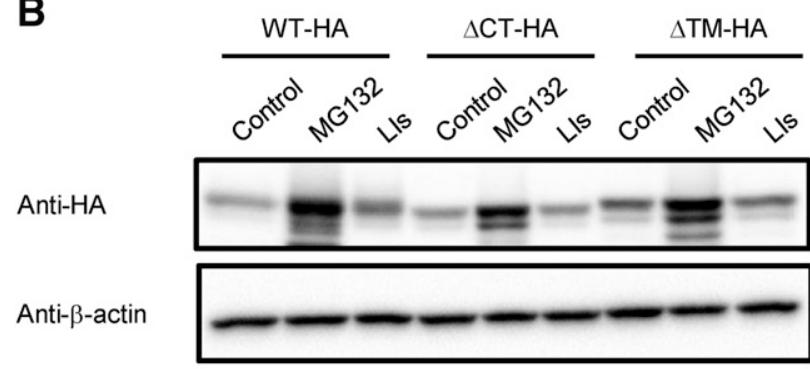

C

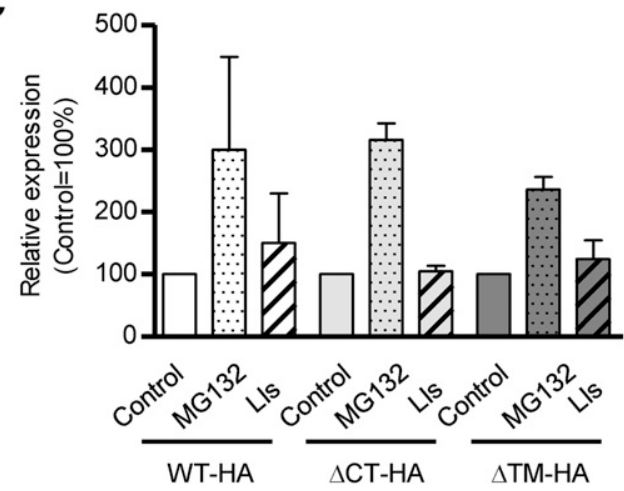

D

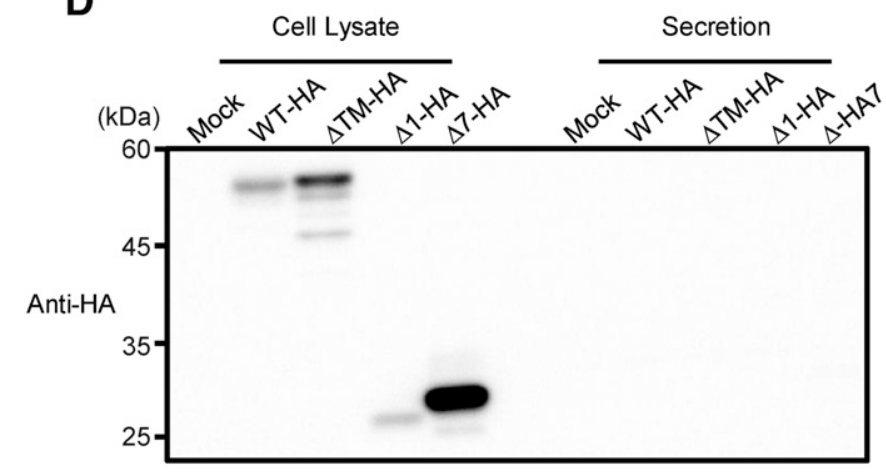

Anti-CD4

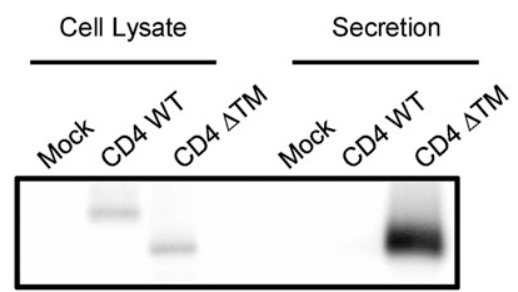

Fig. 7. Analysis of stability and secretion of the UGT2B7-HA mutants. (A) Triton X-100 treatment of microsomal fractions to detect aggregated forms of UGT2B7-HA and its mutants. Homogenates (Homo) were prepared from COS-1 cells with a syringe equipped with a 23 -gauge needle followed by lowspeed centrifugation. An aliquot of homogenate was further fractionated by ultracentrifugation, and supernatants and pellets were collected as cytosols (Cyt) and microsomes, respectively. Microsomes were solubilized in 1\% Triton X-100, and were separated into soluble fraction (Sol.) and insoluble pellet (ppt) by another ultracentrifugation. Homogenate $(15 \mu \mathrm{g})$ and equivalent volumes of the later fractions were analyzed by immunoblotting. Top and bottom panels show the results in short and long exposures, respectively. (B) Effect of treatment with proteasome inhibitor (MG132) and lysosome inhibitor cocktail (LIs) on expression levels of UGT2B7-HAs. COS-1 cells transfected with UGT2B7-HA and its deletion mutants ( $\Delta$ CT and $\Delta$ TM) were treated with $1 \mu \mathrm{M}$ MG132 or LIs, $10 \mu \mathrm{g} / \mathrm{ml} \mathrm{E}-64 \mathrm{~d}, 20 \mu \mathrm{g} / \mathrm{ml}$ leupeptin, and $10 \mu \mathrm{g} / \mathrm{ml}$ pepstatin A for 12 hours. Whole-cell lysates were prepared, and $20 \mu \mathrm{g}$ were analyzed in immunoblotting. (C) The experiments with the inhibitors were performed and quantified three times. Each bar represents the mean \pm S.D. of relative intensities as each control $=100 \%$. (D) Secretion of UGT2B7-HA, CD4, and their deletion mutants were analyzed. COS-1 cells were transfected with each construct and were cultured in FBS-free medium for 12 hours. Cells were harvested for lysate preparation, and medium were concentrated with trichloroacetic acid. The samples were analyzed by immunoblotting with anti-HA antibody and anti-CD4 as primary antibodies.

and -14 by adding an HA-tag to the C-terminus, localized to the ER (Fig. 3B), suggest that the C-terminal DM of UGT2B7 is unnecessary for its ER localization, supporting previous reports (Meech et al., 1996; Meech and Mackenzie, 1997;
Ouzzine et al., 1999). Of note, there are ER-located splice variants of human UGT, which lack the TM and cytoplasmic domains (Girard et al., 2007; Lévesque et al., 2007; Bellemare et al., 2010; Guillemette et al., 2014; Rouleau et al., 2014). 
TABLE 1

The ER retention sites predicted for UGT isoforms

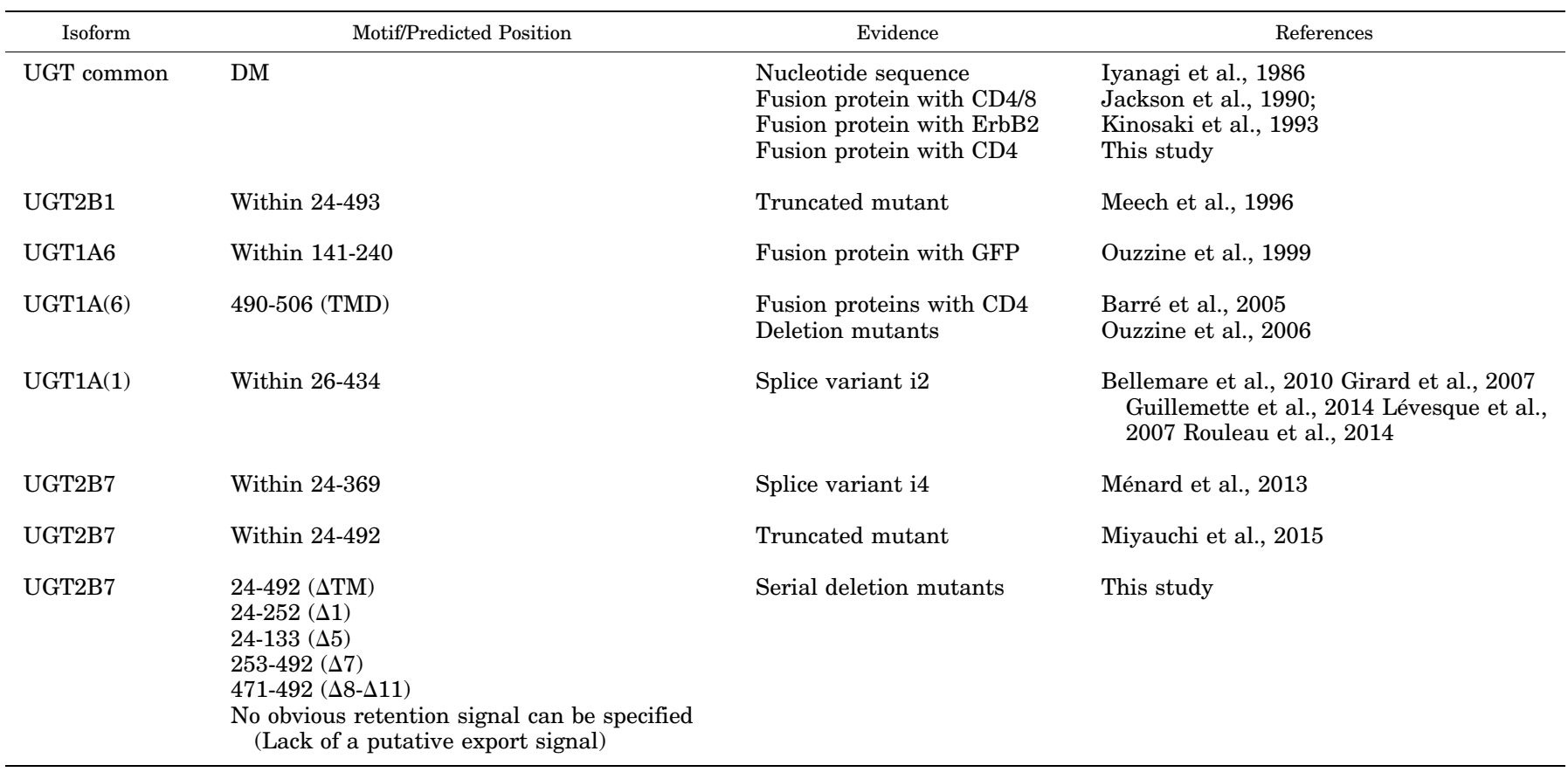

TMD, TM domain.

Similar variants of UGT2B7 showed ER localization despite lacking the DM (Ménard et al., 2013). These findings also reinforce our conclusion that the cytoplasmic region including the $\mathrm{DM}$ is not required for $\mathrm{ER}$ retention.

In contrast, previous studies have brought attention to the role of the DM as a retrieval signal for UGT. Transplantation of the motif altered subcellular localizations of some plasma membrane proteins to the ER (Jackson et al., 1990; Kinosaki et al., 1993). Moreover, it was reported that the interaction of rat UGT2B1 with $\beta$-COP, a subunit in the COPI complex, was abolished by deletion of the C-terminal cytoplasmic tail of UGT2B1 (Meech and Mackenzie, 1998). Given that UGT2B7 is statically retained in the ER, what is the physiologic role of the C-terminal region containing the DM? One possibility is to retrieve UGT2B7 that has escaped to post-ER compartments via nonspecific transport known as "bulk flow." Details for the transport of proteins from the ER by bulk flow remain unclear (Barlowe and Helenius, 2016). Retrieval mediated by the DM should return UGT2B7 to the ER against such a leak mechanism. The importance of this region on catalytic activity was also reported with another UGT isoform. The activity of a water-soluble UGT1A9 mutant lacking the TM and cytoplasmic domains (UGT1A9sol), which were purified from an insect cell expression system, was one order of magnitude lower than microsomal WT UGT1A9 (Kurkela et al., 2004). In addition, we have reported previously that the C-terminal region of UGT2B7 is important for its functional interaction with CYP3A4 (Miyauchi et al., 2015). UGT2B7 WT significantly suppressed CYP3A4 activity, but this suppression disappeared with $\Delta \mathrm{CT}$ and $\Delta \mathrm{TM}$. These lines of evidence clearly indicate a novel role for the UGT C-terminal region as a domain regulating UGT activity and the activity of other drug-metabolizing enzymes, which may improve our understanding of the large interindividual differences in the in vivo catalytic activity of UGT and cytochrome P450 (Shimada et al., 1994; Lamba et al., 2002; Court, 2010).

ER controls the quality of the synthesized proteins, and unfolded and misfolded proteins are degraded by ER-associated degradation (Nakatsukasa and Brodsky, 2008). In this study, we showed that all UGT mutants were predominantly collected in the soluble fraction, thereby suggesting that they were folded correctly (Fig. 7A). Moreover, there was no difference in protein stability among WT and the mutants. These results demonstrated that the ER localization of the mutants is not due to protein misfolding.

Serial mutation analyses suggest that retention or retrieval signals are not needed for ER localization of UGT2B7 in this study. Although many ER membrane proteins do not bear a $\mathrm{DM}$ in their C-terminal ends, they are positioned in the ER,

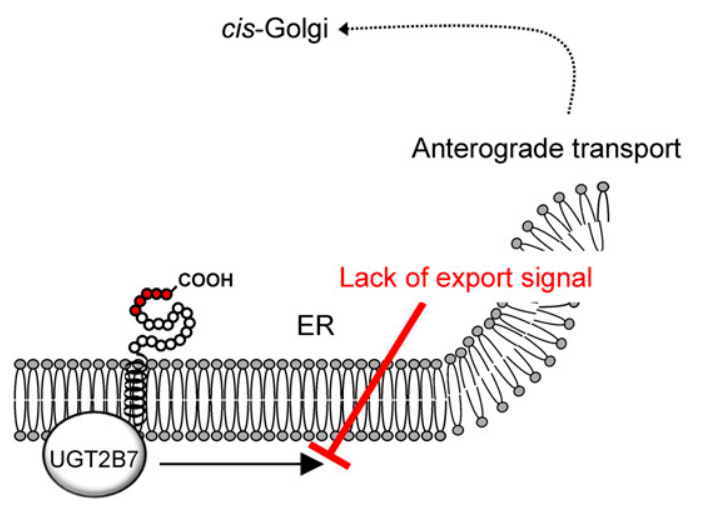

Fig. 8. Predicted mechanism underlying static retention of UGT2B7 in the ER. Lack of an export signal functions as a retention system excluding UGT2B7 from anterograde transport. 
indicating the presence of another mechanism underlying ER localization of membrane protein (Teasdale and Jackson, 1996). One postulated mechanism is that the proteins should have a novel retrieval/retention signal. Previous studies have suggested the presence of ER retention signals of UGT isoforms in either the luminal domain or the cytoplasmic and TM domains (Table 1). However, all of the UGT2B7 deletion mutants we have constructed were observed in the ER without being secreted (Fig. 7D). Thus, it seems more reasonable to assume that UGT2B7 lacks an active signal required for exit from the $\mathrm{ER}$, which results in passive and static retention in the ER (Fig. 8), although little is known about such an export signal.

Further studies are necessary to clarify the molecular mechanism underlying UGT localization in the ER, which may improve our understanding of how ER membrane proteins reside in their respective membrane compartments without retention and/or retrieval motifs.

\section{Acknowledgments}

The authors thank the Research Support Center, Graduate School of Medical Sciences, Kyushu University, for technical support.

\section{Authorship Contribution}

Participated in research design: Miyauchi, S. Kimura, A. Kimura, Kurohara, Hirota, Fujimoto, Mackenzie, Tanaka, Ishii.

Conducted experiments: Miyauchi, S. Kimura, A. Kimura, Kurohara, Hirota.

Performed data analysis: Miyauchi, S. Kimura, A. Kimura, Kurohara, Hirota, Fujimoto.

Wrote or contributed the writing of the manuscript: Miyauchi, Mackenzie, Tanaka, Ishii.

\section{References}

Barlowe C and Helenius A (2016) Cargo capture and bulk flow in the early secretory pathway. Annu Rev Cell Dev Biol 32:197-222.

Barré L, Magdalou J, Netter P, Fournel-Gigleux S, and Ouzzine M (2005) The stop transfer sequence of the human UDP-glucuronosyltransferase 1A determines localization to the endoplasmic reticulum by both static retention and retrieval mechanisms. FEBS J 272:1063-1071.

Bellemare J, Rouleau M, Harvey M, and Guillemette C (2010) Modulation of the human glucuronosyltransferase UGT1A pathway by splice isoform polypeptides is mediated through protein-protein interactions. $J$ Biol Chem 285:3600-3607.

Bock KW (2012) Human UDP-glucuronosyltransferases: feedback loops between substrates and ligands of their transcription factors. Biochem Pharmacol $\mathbf{8 4}$ $1000-1006$

Bowalgaha K, Elliot DJ, Mackenzie PI, Knights KM, and Miners JO (2007) The glucuronidation of $\Delta 4$-3-Keto C19-and C21-hydroxysteroids by human liver microsomal and recombinant UDP-glucuronosyltransferases (UGTs): $6 \alpha-$ and 21-hydroxyprogesterone are selective substrates for UGT2B7. Drug Metab Dispos 35:363-370.

Chowdhury JR, Novikoff PM, Chowdhury NR, and Novikoff AB (1985) Distribution of UDPglucuronosyltransferase in rat tissue. Proc Natl Acad Sci USA 82:2990-2994.

Court MH (2010) Interindividual variability in hepatic drug glucuronidation: studies into the role of age, sex, enzyme inducers, and genetic polymorphism using the human liver bank as a model system. Drug Metab Rev 42:209-224.

Evans WE and Relling MV (1999) Pharmacogenomics: translating functional genomics into rational therapeutics. Science 286:487-491.

Fujimoto K, Ida H, Hirota Y, Ishigai M, Amano J, and Tanaka Y (2015) Intracellular dynamics and fate of a humanized anti-interleukin-6 receptor monoclonal antibody, tocilizumab. Mol Pharmacol 88:660-675.

Gaynor EC, te Heesen S, Graham TR, Aebi M, and Emr SD (1994) Signal-mediated retrieval of a membrane protein from the Golgi to the ER in yeast. J Cell Biol 127: 653-665.

Girard H, Lévesque E, Bellemare J, Journault K, Caillier B, and Guillemette C (2007) Genetic diversity at the UGT1 locus is amplified by a novel 3' alternative splicing mechanism leading to nine additional UGT1A proteins that act as regulators of glucuronidation activity. Pharmacogenet Genomics 17: 1077-1089.

Gomez-Navarro N and Miller E (2016) Protein sorting at the ER-Golgi interface. J Cell Biol 215:769-778.

Guillemette C, Lévesque É, and Rouleau M (2014) Pharmacogenomics of human uridine diphospho-glucuronosyltransferases and clinical implications. Clin Pharmacol Ther 96:324-339.
Harding D, Wilson SM, Jackson MR, Burchell B, Green MD, and Tephly TR (1987) Nucleotide and deduced amino acid sequence of rat liver 17 beta-hydroxysteroid UDP-glucuronosyltransferase. Nucleic Acids Res 15:3936.

Hirota Y and Tanaka Y (2009) A small GTPase, human Rab32, is required for the formation of autophagic vacuoles under basal conditions. Cell Mol Life Sci 66:2913-2932.

Imai Y, Soda M, Inoue H, Hattori N, Mizuno Y, and Takahashi R (2001) An unfolded putative transmembrane polypeptide, which can lead to endoplasmic reticulum stress, is a substrate of Parkin. Cell 105:891-902.

Ishii Y, Koba H, Kinoshita K, Oizaki T, Iwamoto Y, Takeda S, Miyauchi Y, Nishimura Y, Egoshi N, Taura F, et al. (2014) Alteration of the function of the UDP-glucuronosyltransferase 1A subfamily by cytochrome P450 3A4: different susceptibility for UGT isoforms and UGT1A1/7 variants. Drug Metab Dispos 42:229-238.

Iyanagi T, Haniu M, Sogawa K, Fujii-Kuriyama Y, Watanabe S, Shively JE, and Anan KF (1986) Cloning and characterization of cDNA encoding 3-methylcholanthrene inducible rat mRNA for UDP-glucuronosyltransferase. J Biol Chem 261:15607-15614.

Jackson MR, Nilsson T, and Peterson PA (1990) Identification of a consensus motif for retention of transmembrane proteins in the endoplasmic reticulum. EMBO $J$ 9:3153-3162.

Jackson MR, Nilsson T, and Peterson PA (1993) Retrieval of transmembrane proteins to the endoplasmic reticulum. J Cell Biol 121:317-333.

Kinosaki M, Masuko T, Sogawa K, Iyanagi T, Yamamoto T, Hashimoto Y, and FujiiKuriyama Y (1993) Intracellular localization of UDP-glucuronosyltransferase expressed from the transfected cDNA in cultured cells. Cell Struct Funct 18:41-51.

Kurkela M, Mörsky S, Hirvonen J, Kostiainen R, and Finel M (2004) An active and water-soluble truncation mutant of the human UDP-glucuronosyltransferase 1A9. Mol Pharmacol 65:826-831.

Lamba JK, Lin YS, Schuetz EG, and Thummel KE (2002) Genetic contribution to variable human CYP3A-mediated metabolism. Adv Drug Deliv Rev 54:1271-1294

Lévesque E, Girard H, Journault K, Lépine J, and Guillemette C (2007) Regulation of the UGT1A1 bilirubin-conjugating pathway: role of a new splicing event at the UGT1A locus. Hepatology 45:128-138.

Lowry OH, Rosebrough NJ, Farr AL, and Randall RJ (1951) Protein measurement with the Folin phenol reagent. J Biol Chem 193:265-275.

Mackenzie PI, Hjelmeland LM, and Owens IS (1984) Purification and immunochemical characterization of a low-pI form of UDP glucuronosyltransferase from mouse liver. Arch Biochem Biophys 231:487-497.

Meech R and Mackenzie PI (1997) Structure and function of uridine diphosphate glucuronosyltransferases. Clin Exp Pharmacol Physiol 24 907-915.

Meech R and Mackenzie PI (1998) Determinants of UDP glucuronosyltransferase membrane association and residency in the endoplasmic reticulum. Arch Biochem Biophys 356:77-85.

Meech R, Yogalingam G, and Mackenzie PI (1996) Mutational analysis of the carboxy-terminal region of UDP-glucuronosyltransferase 2B1. DNA Cell Biol 15:489-494.

Ménard V, Collin P, Margaillan G, and Guillemette C (2013) Modulation of the UGT2B7 enzyme activity by C-terminally truncated proteins derived from alternative splicing. Drug Metab Dispos 41:2197-2205.

Miyauchi Y, Nagata K, Yamazoe Y, Mackenzie PI, Yamada H, and Ishii Y (2015) Suppression of cytochrome P450 3A4 function by UDP-glucuronosyltransferase 2B7 through a protein-protein interaction: cooperative roles of the cytosolic carboxyl-terminal domain and the luminal anchoring region. Mol Pharmacol 88:800-812.

Munro S and Pelham HR (1987) A C-terminal signal prevents secretion of luminal ER proteins. Cell 48:899-907.

Nakatsukasa K and Brodsky JL (2008) The recognition and retrotranslocation of misfolded proteins from the endoplasmic reticulum. Traffic 9:861-870.

Nilsson T, Jackson M, and Peterson PA (1989) Short cytoplasmic sequences serve as retention signals for transmembrane proteins in the endoplasmic reticulum. Cell 58:707-718.

Ouzzine M, Barré L, Netter P, Magdalou J, and Fournel-Gigleux S (2006) Role of the carboxyl terminal stop transfer sequence of UGT1A6 membrane protein in ER targeting and translocation of upstream lumenal domain. FEBS Lett 580:1953-1958.

Ouzzine M, Magdalou J, Burchell B, and Fournel-Gigleux S (1999) An internal signal sequence mediates the targeting and retention of the human UDPglucuronosyltransferase $1 \mathrm{~A} 6$ to the endoplasmic reticulum. $J$ Biol Chem 274:31401-31409

Pääbo S, Bhat BM, Wold WS, and Peterson PA (1987) A short sequence in the COOHterminus makes an adenovirus membrane glycoprotein a resident of the endoplasmic reticulum. Cell 50:311-317.

Pääbo S, Weber F, Nilsson T, Schaffner W, and Peterson PA (1986) Structural and functional dissection of an MHC class I antigen-binding adenovirus glycoprotein. EMBO J 5:1921-1927.

Pelham HR (1988) Evidence that luminal ER proteins are sorted from secreted proteins in a post-ER compartment. EMBO $J$ 7:913-918.

Radominska-Pandya A, Czernik PJ, Little JM, Battaglia E, and Mackenzie PI (1999) Structural and functional studies of UDP-glucuronosyltransferases. Drug Metab Rev 31:817-899.

Radominska-Pandya A, Ouzzine M, Fournel-Gigleux S, and Magdalou J (2005) Structure of UDP-glucuronosyltransferases in membranes. Methods Enzymol 400:116-147.

Rouleau M, Roberge J, Bellemare J, and Guillemette C (2014) Dual roles for splice variants of the glucuronidation pathway as regulators of cellular metabolism. $\mathrm{Mol}$ Pharmacol 85:29-36. 
Rowland A, Miners JO, and Mackenzie PI (2013) The UDP-glucuronosyltransferases: their role in drug metabolism and detoxification. Int $J$ Biochem Cell Biol 45:1121-1132.

Schrader M, Godinho LF, Costello JL, and Islinger M (2015) The different facets of organelle interplay-an overview of organelle interactions. Front Cell Dev Biol 3:56.

Shepherd SR, Baird SJ, Hallinan T, and Burchell B (1989) An investigation of the transverse topology of bilirubin UDP-glucuronosyltransferase in rat hepatic endoplasmic reticulum. Biochem J 259:617-620

Shim SH (2017) Cell imaging: an intracellular dance visualized. Nature 546:39-40.

Shimada T, Yamazaki H, Mimura M, Inui Y, and Guengerich FP (1994) Interindividual variations in human liver cytochrome P-450 enzymes involved in the oxidation of drugs, carcinogens and toxic chemicals: studies with liver microsomes of 30 Japanese and 30 Caucasians. J Pharmacol Exp Ther 270:414-423.
Teasdale RD and Jackson MR (1996) Signal-mediated sorting of membrane proteins between the endoplasmic reticulum and the golgi apparatus. Annu Rev Cell Dev Biol 12:27-54

Ziegler K, Tumova S, Kerimi A, and Williamson G (2015) Cellular asymmetric catalysis by UDP-glucuronosyltransferase $1 \mathrm{~A} 8$ shows functional localization to the basolateral plasma membrane. J Biol Chem 290:7622-7633.

Address correspondence to: Yuu Miyauchi, Division of Pharmaceutical Cell Biology, Graduate School of Pharmaceutical Sciences, Kyushu University, 3-11 Maidashi, Higashi-ku, Fukuoka 812-8582, Japan. E-mail: ymiyauchi@phar. kyushu-u.ac.jp; or Yuji Ishii, Laboratory of Molecular Life Sciences, Graduate School of Pharmaceutical Sciences, Kyushu University, 3-1-1 Maidashi, Higashiku, Fukuoka 812-8582, Japan. E-mail: ishii@phar.kyushu-u.ac.jp 